\title{
The molecular basis of mutagenesis -40 years of research on genomic integrity
}

\author{
E C Friedberg, BSc, MB BCh, FRC (Path) \\ Errol Friedberg works in the Laboratory of Molecular Pathology, Department of Pathology, University of Texas Southwestern Medical Center \\ at Dallas, Dallas, Texas, USA.
}

Corresponding author: E C Friedberg (errol.friedberg@utsouthwestern.edu)

This article reviews the multiple mechanisms by which both prokaryotes and eukaryotes remove or tolerate various types of naturally occurring and exogenously generated base damage in DNA.

S Afr Med J 2013;103(12 Suppl 1):982-983. DOI:10.7196/SAMJ.6932

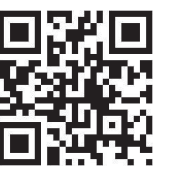

\section{Excision repair of} damaged DNA

In the mid-1960s the prevalent model for excision repair in bacteria implicated some sort of enzymatic activity that specifically recognises the presence of damaged nucleotides in DNA and incises the damaged DNA strand close to the site of damage, thereby facilitating subsequent excision of a fragment of DNA containing the offending nucleotide. Years of work failed to uncover the putative enzyme in extracts of bacteria that specifically recognise base damage generated by the exposure of cells to ultraviolet (UV) radiation, a naturally occurring mode of DNA damage associated with exposure of cells in the skin to sunlight.

However, after turning to extracts of bacteria infected with a bacteriophage called T4, I identified such an enzyme activity. Further studies, performed mainly at Stanford University, revealed that the phage T4 enzymes specifically and uniquely incise DNA containing photoproducts called pyrimidine dimers. Further work in my laboratory and by others demonstrated that the phage enzyme was a rare and exotic 'bird' encountered in very few organisms. Nonetheless, deciphering the precise mode by which this type of enzyme cuts damaged DNA revealed that it is not restricted to DNA containing pyrimidine dimers. Multiple such enzymes (called DNA glycosylases) recognise and cut DNA containing different types of damage. These enzymes are present in organisms ranging from bacteriophages to humans. Furthermore, it

Much of Professor Trefor Jenkins' professional life has dealt with the study of genetics and genes, topics that he and I have spent countless hours discussing over the years, especially since my own research interests lie in the area of DNA damage and repair. This article is dedicated to Trefor's long and fruitful career as a population geneticist, and summarises my own research in the field of DNA repair over the past 40 years. I was introduced to the topic while completing two years of postdoctoral training with the late David A Goldthwait in the Department of Biochemistry at Case Western University in Cleveland, Ohio. In the course of this experience and during two years I spent at the Walter Reed Army Institute of Research, I sought to understand some of the mysteries of a DNA repair process called excision repair, a process discovered in the 1960s, by which multiple types of damaged or inappropriately positioned nucleotides in DNA are excised from the genome. was shown by others that all DNA glycosylases initiate the excision of certain types of base damage by removal of the free base. This process is therefore called base excision repair. In the interim the molecular mechanism of an alternative and more general mode of excision repair of the type I was initially in search of was unravelled by others. This process, widespread in nature, is called nucleotide excision repair (NER). Several investigators subsequently established that humans suffering from the rare autosomal recessive disease xeroderma pigmentosum (XP) are defective in NER and consequently suffer an enormously increased risk of developing skin cancer following exposure to sunlight. As a trained pathologist, my interest in understanding the molecular basis of XP prompted me to shift my research to a eukaryotic model, the yeast Saccharomyces cerevisiae. This transpired at a time when gene cloning was coming into its own, and after isolating a series of yeast mutants defective in NER my colleagues and I revealed a requirement for multiple distinct genes, indicating a profound biochemical complexity of NER in higher organisms.

With multiple yeast genes in hand we were also able to show that these are evolutionarily conserved in humans, facilitating both the molecular cloning of multiple human XP genes and comprehension of the enormous biochemical complexity of NER in higher organisms. Of particular note was a discovery in my laboratory and by others that some of these genes are essential for DNA transcription by RNA polymerase. This discovery provided important insights into the observation that individuals carrying mutations in certain XP genes are prone to disease states such as Cockayne syndrome and trichothiodystropy, which may be explained by defective transcription.

\section{Xeroderma pigmentosum}

We additionally demonstrated that inactivation of any XP genes in mice (using conventional gene knock-out technology) results in skin disease, including a markedly increased predisposition to cancer of the shaved skin following daily exposure to UV radiation. We also observed that these mutant mice were prone to liver cancer when exposed to chemicals known to promote neoplasia when fed to experimental animals. This observation strongly suggests that humans suffering from XP are probably prone to cancer in organs other than the skin given sufficient exposure to chemical carcinogens - a result to be expected, based on the observation that NER deals with multiple types of base damage in DNA, not just pyrimidine dimers.

Most prokaryotes and eukaryotes are endowed with other mechanisms for removing (excising) damaged or inappropriate bases from 
DNA. As mentioned above, one class of enzymes catalyse the release of the offending base as the free base. Another class of repair enzymes specifically excises mismatched bases (that generally arise from replicative errors by high-fidelity enzymes) and defines a third mode of excision repair called mismatch repair.

All dividing cells contain one or more high-fidelity DNA polymerases that are responsible for accurate replication of the genome. However, high-fidelity DNA polymerases are unable to replicate template strands of DNA containing most, if not all, altered bases, resulting in replicative arrest. About 10 years ago several laboratories, including my own, discovered a novel class of specialised DNA polymerases that are able to do so via a process referred to as translesion DNA synthesis (TLS). Consonant with this property, these specialised DNA polymerases have extremely low fidelity when copying undamaged DNA, i.e. their ability to replicate past sites of damage is a function of their extremely low fidelity when copying any DNA. This and other mechanisms that can overcome replicative arrest associated with base damage belong to a cellular response to DNA damage called DNA damage tolerance, so named because the potentially lethal consequences of replicative arrest are mitigated without removal (excision) of damaged or inappropriate bases.

\section{DNA damage tolerance}

Bacteria such as Escherichia coli are endowed with three specialised DNA polymerases. However, many eukaryotes carry several such enzymes - indeed, mammalian (including human) cells are endowed with no less than 10 . Hence, among the many questions currently being addressed about these interesting enzymes is the following: Why do mammalian cells contain so many specialised DNA polymerases?

One possible answer derives from experiments indicating that some of these enzymes may have evolved to promote for the mutational changes that are associated with antibody maturation. However, some lower eukaryotes that evolved before the emergence of immunity also possess specialised polymerases, indicating that they did not all evolve primarily to support somatic hypermutation or class switching.

Another tenable explanation for the presence of multiple specialised DNA polymerases in vertebrates is that each evolved to accurately bypass a particular type of naturally occurring base damage. This is supported by the observation that one of these enzymes, DNA polymerase eta (Pol $\eta)$, supports accurate DNA replication past pyrimidine dimers in vitro. There is compelling evidence that this also transpires in vivo. Specifically, it has been known for many years that $100 \%$ of patients with prototypic clinical features of XP are proficient in NER. These patients are therefore classified as belonging to the variant form of XP. Remarkably, all XP variant patients examined so far have been shown to carry inactivating mutations in the gene that encodes Pol $\eta$.

This intriguing finding leads to the hypothesis that the Polh gene, which encodes Pol $\eta$, evolved specifically to relieve replicative arrest associated with the presence of unexcised pyrimidine dimers in DNA following exposure of cells to UV radiation - and does so accurately. In the absence of functional Pol $\eta$, one or more other specialised DNA polymerases presumably subserves this function, thereby avoiding potential replicative arrest and subsequent cell death, but at the cost of acquiring a mutational burden.

If Pol $\eta$ did in fact evolve to accurately replicate past unrepaired pyrimidine dimers, and if this is the exclusive function of this enzyme, one would not expect cells defective in it to manifest mutations in cells exposed to other types of DNA damage. Consistent with this expectation, Polh-defective mouse cells do not manifest an increased frequency of spontaneous mutations in vitro.

Assuming that some if not all of the remaining specialised DNA polymerases in mammalian cells evolved to bypass other particular types of naturally occurring base damage in DNA, the question arises, what sorts of damage might this be?

\section{Mice defective in the Polk gene}

My colleagues and I generated a mouse strain that is mutated in the Polk gene (which encodes DNA polymerase $\mathrm{K}$ ) and have sought to determine the 'cognitive' substrate for this enzyme. In vitro, purified Polk has been shown to bypass a number of different types of base damage in DNA with no obvious structural similarity, and does so with varying degrees of fidelity. Furthermore, Polk-mutant mice do not manifest any obvious phenotype. However, cells from such mice are hypersensitive to killing by the polycyclic aromatic compound benzo[a]pyrene, a well-known carcinogen that is emitted from fuels, fires and cigarette smoke. In contrast to mouse cells defective for Pol $\eta$, Polk cells are spontaneous mutators, suggesting that Polk is indeed required for the replicative bypass of some type(s) of spontaneous DNA damage.

Benzo[a]pyrene is not widely distributed in nature. Hence, our current working hypothesis is that the 'cognitive' substrate for Polk may be a naturally occurring polycyclic compound(s). We have focused our attention on cholesterol and the many cholesterol derivatives generated during the biosynthesis and degradation of polycyclic hormones such as oestrogen, progesterone, testosterone and glucocorticoids.

In recent experiments we have sought to make wild-type and Polkmutant mice hypercholesterolaemic by feeding them high-cholesterol diets. After some months of this protocol, and failing to observe raised serum levels of cholesterol, we realised that mice cannot be rendered hypercholesterolaemic unless they are defective in either the $A p o E$ gene or a gene called $L D L R$ (for low-density lipoprotein (LDL) receptor). After generating Polk $k^{-} A$ poE $E^{-/}$and Polk ${ }^{-/} L L^{-/}$double-mutant mice, significant hypercholesterolaemia was observed (W D Singer and E C Friedberg unpublished data).

Preliminary experiments carried out to date have demonstrated a significantly enhanced frequency of mutations in cells from the liver, spleen, kidneys and lungs (the only organs studied so far) in both doublemutant combinations. Examination of the mutational spectra in these mutant tissues reveals a distinct preponderance of G:C transversions, an observation consistent with the fact that polycyclic aromatic compounds bind primarily to the $\mathrm{N} 2$ position of guanine, causing replicative mispairing that converts $\mathrm{G}: \mathrm{C}$ base pairs to mutant base pairs.

At the time of writing, cohorts of mice that are defective in the ApoE or LDLR genes mentioned above, and are either Polk $k^{+++}$or Polk a high-cholesterol diet and being monitored closely for signs of disease.

In addition to their presence in somatic cells, specialised DNA polymerases are expressed in the testis (and possibly in ova). The enhanced mutagenesis potentially associated with TLS in germ-line cells may play an important role in generating mutations that help drive the genetic heterogeneity required for Darwinian evolution.

DNA damage tolerance mechanisms, i.e. biochemical pathways other than TLS, have also been discovered. These are uniformly associated with enhanced mutagenesis.

\section{Further reading}

Readers interested in learning more about DNA repair and mutagenesis are referred to: - Friedberg EC, Walker GC, Siede W, et al. DNA Repair and Mutagenesis. 2nd ed. ASM Press, 2007.

\section{The following review articles may also be consulted:}

- Robertson AB, Klungland A, Rognes T, Leiros I. DNA repair in mammalian cells: Base excision repair: The long and short of it. Cell Mol Life Sci 2009;66(6):981-993. [http://dx.doi.org/10.1007/s00018-009-8736-z] McCullough, AK, Dodson ML, Lloyd SR. Initiation of base excision repair: Glycosylase mechanisms an structures. Annu Rev Biochem 1999;68(1):255-285. [http://dx.doi.org/10.1146/annurev.biochem.68.1.255] 